\title{
Sonic intimacy in the music of Billie Eilish and recordings that induce ASMR
}

\author{
Lindsay Warrenburg, ${ }^{1 \dagger}$ Nathan Centa, ${ }^{1}$ Xintong Li, ${ }^{2}$ Hansae Park, ${ }^{2}$ Diana Sari, ${ }^{1}$ Feiyu Xie ${ }^{2}$ \\ ${ }^{1}$ School of Music, Ohio State University, Columbus, OH, USA \\ ${ }^{2}$ Department of Psychology, Ohio State University, Columbus, OH, USA \\ $\dagger$ Corresponding author: lindsay.a.warrenburg@gmail.com \\ Published 16 December 2021; https://doi.org/10.18061/FDMC.2021.0026 \\ Author video presentation and/or other conference material: https://doi.org/10.17605/OSF.IO/NWZ7Y
}

\begin{abstract}
This article explores similarities in the music of Billie Eilish and recordings that induce the Autonomous Sensory Meridian Response (ASMR). Two complementary approaches are presented. First, the methodology and preliminary results of an empirical study are reported, which investigates peoples' emotional responses to Eilish's music, mouth-related ASMR sounds (oral wetness cues, whispering, breathing sounds), and non-mouth ASMR sounds (tapping, scratching). Second, a new theory of sonic intimacy is presented that draws on similar electroacoustic techniques in the music of Billie Eilish and Bing Crosby and may account for their popularity during times of stress and isolation.
\end{abstract}

KEYWORDS: Billie Eilish, ASMR, emotion, sonic intimacy, musicovid

\section{Introduction}

Billie Eilish is a popular singer who rose to fame with her 2019 album WHEN WE ALL FALL ASLEEP, WHERE DO WE GO?. At the age of 17, she became the first Gen Z musician to hit No. 1 on the Billboard charts, where her single bad guy was in the Top 100 list for 49 weeks, with more than 25 of those weeks in the Top 10 . Eilish is the youngest person to win the 4 major Grammys in the same year, in 2019 winning Best New Artist, Record of the Year, Song of the Year, and Album of the Year, as well as Best Pop Vocal Album. Although iTunes classifies her music as "alternative," she describes her music as "genre-less" (Crozier, 2019).

The beginning of the music video for bad guy gives a taste of Billie's music: The video starts by focusing on saliva sounds as she takes out her Invisalign. Table 1 lists some of the techniques at which critics and audiences have marveled, including the use of close miking, breathing sounds, whispering, binaural effects, and the use of dentist drills and Easy-Bake Ovens (e.g., Connick, 2019).

Researchers, journalists, and audience members have noted that there are similar electroacoustic effects in Eilish's music and audiovisual recordings that induce ASMR-Autonomous Sensory Meridian Response (e.g., Geaghan-Breiner \& Gmoser, 2019). ASMR is a sensory response to certain auditory stimuli, which includes tingling and static-like sensations across the body, as well as feelings of relaxation, wellbeing, and psychological flow (Barratt, Spence, \& Davis, 2017). Videos and sounds that evoke ASMR are extremely popular among Millennials and Gen $Z$ listeners: As of February 2021, the YouTube channel "Gentle Whispering ASMR" has 2 million subscribers and 846 million views, and the Reddit page "ASMR. Sounds that feel good" has 229,000 members.

Sounds that elicit ASMR include whispering, speaking with less vocal energy, slow speaking rates, increased use of pauses, oral wetness cues, the greater use of silibants, sounds of breathing, alternating binaural stimuli, crisp sounds, and tapping or scratching sounds (Andersen, 2015; Kovacevich \& Huron, 2019). Broadly, ASMR-inducing clips can be broken into two categories - mouth-related sounds and non-mouth sounds. As might be expected, mouth-related sounds include features like audible breathing sounds or oral wetness cues (e.g., sounds of saliva), whereas nonmouth sounds include effects such as tapping or crinkling paper.

This article explores whether the popularity of ASMRtists and Billie Eilish can be attributed, in part, to shared sonic techniques that elicit feelings of intimacy and close physical proximity. First, the methodology and preliminary results of an empirical study will be described. Second, a new theory, called sonic intimacy, is proposed, which may account for the popularity of certain artists and genres during times of isolation and loneliness, including the current coronavirus pandemic.

Table 1: Some audio effects in Billie Eilish's music.

\begin{tabular}{|l|l|c|}
\hline \multicolumn{1}{|c|}{ Effect } & \multicolumn{1}{c|}{ Track } & Timing \\
\hline Close miking & goodbye & $00: 00-00: 30$ \\
\hline Whispering & goodbye & $01: 18-01: 48$ \\
\hline Binaural effects & bury a friend & $01: 04-01: 13$ \\
\hline Dentist drills & bury a friend & $01: 51-02: 00$ \\
\hline
\end{tabular}




\section{Methods}

\section{Stimulus Selection}

A team of researchers listened to Billie Eilish's album WHEN WE ALL FALL ASLEEP, WHERE DO WE GO? and to the three most popular albums on iTunes under the search term "ASMR": (1) FrivolousFox's ASMR Lollipop Trigger Words \& Up Close Mouth Sounds, (2) ASMR HeadHI, Asmr \& Asmr Sleep Sounds's Assorted Asmr: Slime, Tapping \& Whispers, and (3) GiBi ASMR's Extreme Secret Whispering. From the complete recordings, passages were selected that best corresponded to published features of ASMR (Andersen, 2015; Kovacevich \& Huron, 2019).

The resulting list of excerpts included 22 passages by Billie Eilish and 62 passages from an ASMR-designated album. Each member of a six-person research team rated a random subset of stimuli on certain criteria (described below). In order to better enable within-stimulus group comparisons, half of the researchers were randomly selected to rate Billie Eilish passages before ASMR passages; the other three researchers rated ASMR passages before Billie Eilish passages.

Each of the Billie Eilish passages was rated by two or three researchers on two criteria: (1) the percent of the clip that contained ASMR-related features $(0$ : very little ASMR, 100: exclusively ASMR) and (2) its overall quality as a stimulus, with regard to duration, loudness, and the inclusion of lyrics (0: very poor quality, 100: very high quality). An average "ASMR score" and "quality score" was calculated for each excerpt. The ASMR scores ranged from 36.6 to $86.6(\mathrm{M}=75.8, \mathrm{SD}$ $=13.1)$ and quality scores ranged from 33.3 to $88.3(\mathrm{M}$ $=76.8, \mathrm{SD}=10.8$ ). The 22 excerpts were then sorted by average quality score, followed by average ASMR score. The top ten excerpts from this ranked list were used as the final Billie Eilish stimuli. The length of these excerpts ranged from 5 to 56 seconds in duration. Because the experiment location was the local science museum, an a priori decision was made not to use any recording longer than 30 seconds. Therefore, for the three long passages, the first 30 seconds were used as the final stimulus. The final Billie Eilish stimuli had an average length of 19 seconds ( $\mathrm{SD}=1$ second).

Similarly, each of the ASMR-inducing passages was rated by two or three researchers on two criteria: (1) its similarity to Billie Eilish's music writ large (0: very little similarity, 100: very high similarity) and (2) its overall quality as a stimulus (0: very poor quality, 100: very high quality). An average "similarity score" and "quality score" was calculated for each excerpt. The similarity scores ranged from 7.5 to $91.7(\mathrm{M}=64.3, \mathrm{SD}$ $=20.2$ ) and the quality scores ranged from 52.5 to 91.7 $(\mathrm{M}=76.2, \mathrm{SD}=10.4)$. The passages were then sorted by average quality score, followed by average similarity score. Passages that included audible words or phrases were excluded. From this ranked list, the top ten "mouth-related" excerpts-ASMR sounds that were made by a person's mouth, such as whispering, tongue clicking, and oral wetness cues - and the top ten "nonmouth" excerpts - ASMR sounds that were not made by a person's mouth, such as tapping, scratching, crinkling, and bubbles - were used as stimuli in the experiment. Once again, the first 30 seconds of each excerpt longer than 30 seconds was used as the final stimulus. The "mouth-related" stimuli had an average length of 11 seconds (SD $=6$ seconds) and the "non-mouth" stimuli had an average length of 16 seconds ( $\mathrm{SD}=9$ seconds).

\section{Experimental Procedure}

The experiment was conducted at the Center of Science and Industry in Columbus, Ohio, USA. Researchers were stationed outside an exhibit and asked entering visitors if they would be interested in participating in a short music-related study. In order to participate, participants were required to be at least 18 years old and could not have a history of speech, language, or hearing problems. The Ohio State University Institutional Review Board \#2019E0809 approved all procedures.

Each participant heard a random selection of six stimuli (out of the 30 total stimuli). A randomizer was programmed to evenly present all 30 items, but there were no controls ensuring that everyone heard the same number of music and non-music stimuli. For each stimulus, participants were asked: While listening to this clip, how much did you experience the following things? (1) tingling, (2) relaxation or calm, (3) discomfort or anxiety, (4) enjoyment. Participants responded to each question on a $0-100$ continuous scale, anchored at 0 (none). They were also asked: Would you listen to this clip again? with the options of yes, maybe, and no.

In addition to these stimulus-related questions, participants were asked whether they had prior experiences of ASMR and synesthesia, as well as their overall level of musicianship (Ollen, 2006), age, preferred gender, race/ethnicity, first language, and zipcode. A series of personality-related questions included four questions from the Social Anxiety Questionnaire for Adults (regarding public teasing, talking to strangers, being asked a question by a superior, and looking in the eyes of a stranger while talking; Caballo et al., 2015), two questions from the 
Big-Five Trait Taxonomy (regarding their curiosity about many different things and their level of value for artistic and aesthetic experiences; John \& Srivastava, 1999), four questions from the Ten-Item Personality Inventory (regarding trait anxiety, openness to new experiences, emotional stability, and conventionality; Gosling, Rentfrow, \& Swann Jr., 2003), and their preference for Dance/Electronica music from the STOMP-R (Rentfrow, Goldberg, \& Levitin, 2011).

\section{Preliminary Results}

Of a planned cohort of 400 participants, only 17 competed the survey before the science museum closed due to COVID-19. Seven participants identified as female, four identified as male, and six did not answer. The average age was 30.5 years (range $=21$ to 40 ).

Because of the small sample size, no statistical tests were conducted. Figure 1 shows violin plots of the amount of anxiety, enjoyment, relaxation, and tingling sensations that participants felt when listening to Eilish's music, mouth-related ASMR sounds, and nonmouth ASMR sounds. Although more data is needed to substantiate these trends, the results could be consistent with the idea that people experience less anxiety and more relaxation and enjoyment when listening to Eilish's music than when listening to ASMR-related sounds.

\section{Discussion}

Certain acoustic characteristics, like whispering and breathing sounds, are characteristics of close physical proximity (Kovacevich \& Huron, 2019). Namely, when we hear sounds like someone breathing, our brain is wired to assume that there is a person physically close to us. Recordings created to elicit ASMR tend to draw on these sonic characteristics, which can result in feelings of intimacy or personal attention (Kovacevich $\&$ Huron, 2019). The current study aimed to test whether people experience similar affective states in response to the proximity-related cues in recordings by ASMRtists and in the music of Billie Eilish. The preliminary results of the current study suggest that even though there are shared sonic techniques in these two domains, people did not enjoy the experience of listening to ASMRrelated recordings as much as they enjoyed the music of Billie Eilish.

Once the COVID-19 lockdown is over, a larger cohort of participants will be used to test for the influence of demographic, health, and personality variables, including social anxiety and extraversion. Future research will also include participants who purposefully seek out ASMR-inducing recordings.
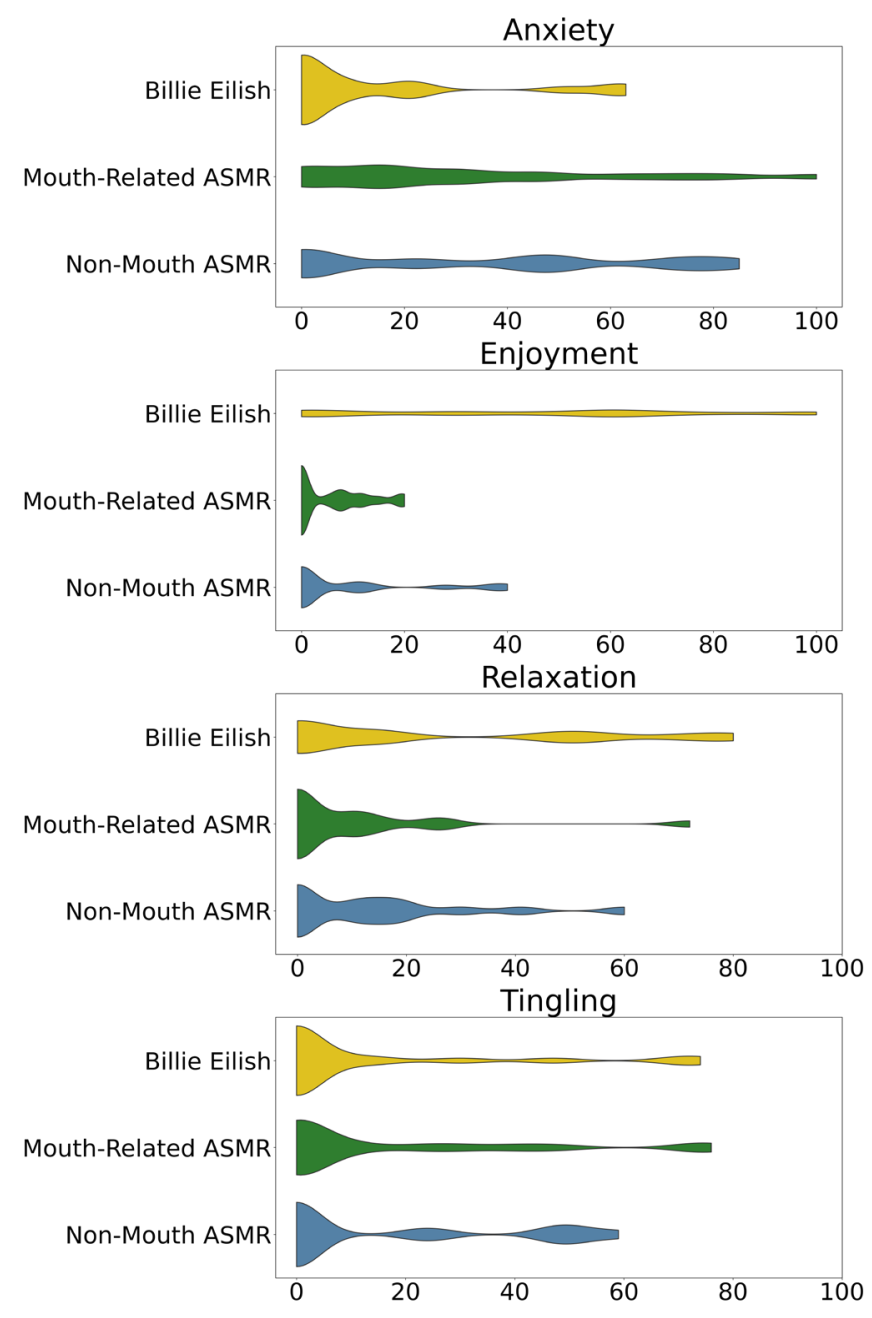

Figure 1: Violin plots of self-reported ratings of anxiety, enjoyment, relaxation, and tingling when listening to the music of Billie Eilish, mouth-related ASMR sounds, and non-mouth ASMR sounds (0: none, 100: high amount).

\section{Theoretical Implications: Sonic Intimacy}

Recordings by Billie Eilish and ASMRtists have soared in popularity among Gen $\mathrm{Z}$ and Millennial listeners. If this popularity can be explained, in part, through shared sonic techniques like close miking and whispering, the question arises if these techniques also contribute to the popularity of other artists. The following section explores the music of the American crooners in the first half of the twentieth century and proposes a theory that may account for the popularity of the crooners, Billie Eilish, and ASMRtists: sonic intimacy. 


\section{Crooning and Bing Crosby}

The recording techniques in the music of Billie Eilish share similarities with the music of the American crooners of the 1930s through the 1950s, such as Bing Crosby. Before the era of the crooners, much of the music that appealed to the American masses was cabaret-style music that involves loudly projecting your voice. Crooning, by contrast, involves quieter and more conversational styles of singing (McCracken, 1999). This crooning style became popular among the American public in the 1920s. The origins of the popular (white) crooning style are steeped in the appropriation of Black music and minstrel songs and by making this music popular among the dominant American culture (McCracken, 1999). Early white crooners, like Al Jolson and Eddie Cantor, even performed in blackface. The American public saw figures like Bing Crosby as "legitimiz[ing] crooning by connecting it to traditional notions of white masculinity: a good work ethic, patriarchy, religious belief, white supremacy, and contained emotion" (McCracken, 1999, p. 389).

Despite these racist origins, the American public embraced Crosby: At the height of his popularity, 25\% of Americans listened to his radio shows and he was voted as the most admired man in America (Barnes, 2001). Crosby was a source of comfort during the Great Depression and World War II, both in America and abroad. Crosby's beloved status lasted throughout his lifetime and he became the first recipient of the Grammy Lifetime Achievement Award in 1963.

McCracken (1999) notes that the development of microphone technologies, such as the ribbon dynamic microphone in 1924, may have helped contribute to the popularity of crooning, as these electronic recordings were able to convey wider frequency ranges and more complex sounds than the previous recordings. While performing, singers could use the improved microphone to convey a more personal tone. This intimate performance style, including close miking and conversational speaking, was Bing Crosby's signature.

\section{Sonic Intimacy}

Recall that certain features tend to induce ASMR: close miking, whispering, audible breathing, and oral wetness cues. These sonic effects may mimic neural cues of physical proximity and may even activate the parasympathetic nervous system (Andersen, 2015; Kovacevich \& Huron, 2019). We propose that these findings extend to the musical domain and, in particular, to the music of Billie Eilish and Bing Crosby. To introduce a new term, moments when music uses techniques like breathing sounds, close miking, whispering, and binaural effects can be described as exhibiting sonic intimacy. Using this definition, the music of Billie Eilish and Bing Crosby contains a multitude of sonically intimate sections (Table 2). When listening to sonically intimate moments, listeners may feel the same sense of relaxation and pleasure as being physically near a loved one.

In support of this idea, some snapshots of history indicate when musicians who use sonic intimacy become exceedingly popular. After the 1929 stock market crash, record sales, vaudeville houses, and nightclubs disappeared for most of the American public (McCracken, 1999). Crosby's use of inventions such as tape-based recording and the electronic microphone helped his popularity soar in the 1930s and 1940s (Hammar, 1989). Billie Eilish rose to fame among Gen $\mathrm{Z}$ listeners in 2019, which could suggest the need for new methods of intimacy after the rise of social media. Of course, the loneliness and isolation felt during the coronavirus crisis would also seem to be a good time to engage with sonically intimate music. Composer Scott Ordway recently described his series of compositions, called "Interiors," that was composed during the coronavirus crisis as works "created in isolation to be performed by musicians in isolation and heard by listeners in isolation. They are quiet, personal, fragile pieces whose medium is the microphone rather than the concert hall. You'll hear creaking piano benches, fingers brushing across clothing.... and the incredible quality of sounds produced at the edge of silence, tone colors which would never be audible in live performance. Music under a microscope" (Ordway, 2020).

\section{Conclusion}

During a time of social distancing and lockdown, such as the COVID-19 pandemic, listeners may turn to sounds that induce feelings of intimacy or closeness, even though the artist or performer may not be physically present. What we have defined as sonic intimacy includes electroacoustic features such as close miking, whispering, oral wetness cues, sounds of breathing, and binaural beats. These techniques are common among recordings that induce the sensory phenomenon of ASMR. We suggest that these moments of sonic intimacy may also contribute to the popularity of artists like Billie Eilish and Bing Crosby, who spoke to audiences nearly 100 years apart. 
Table 2: Sonic intimacy in Eilish and Crosby's music.

\begin{tabular}{|l|l|}
\hline \multicolumn{1}{|c|}{ Artist } & \multicolumn{1}{c|}{ Quotation } \\
\hline Eillish & $\begin{array}{l}\text { She's decisively young and CAREFREE in } \\
\text { that sort of neon bisexual lightning flashy } \\
\text { strobe streetwear instagram-story urgency } \\
\text { without being at all naive or Lolita-sexualized. } \\
\text { ALSO! I think gen z is extremely lonely!!!! } \\
\text { songs like 'When the Party's Over' get } \\
\text { RIGHT at the desperate, gnawing sense of } \\
\text { isolation social media has sewn into a } \\
\text { generation who are becoming increasingly } \\
\text { sequestered (Petrusich, 2019) }\end{array}$ \\
\hline $\begin{array}{l}\text { Billie } \\
\text { Eilish }\end{array}$ & $\begin{array}{l}\text { Everything is close-mic'd and whispered...the } \\
\text { goal is to always have Billie's vocals sound } \\
\text { super-present.' It's as if she's right there, } \\
\text { murmuring directly into your ear (Geaghan- } \\
\text { Breiner \& Gmoser, 2019) }\end{array}$ \\
\hline Billie & $\begin{array}{l}\text { Both oh-so-2019 products of the internet, the } \\
\text { respective rises of tingle-sensation du } \\
\text { jour ASMR and pop prodigy Billie Eilish have } \\
\text { run parallel to each other (Connick, 2019) }\end{array}$ \\
\hline Bing \\
Crosby & $\begin{array}{l}\text { [Crosby treated] a new invention, the } \\
\text { microphone, as if it were a friend's ear...[He] } \\
\text { shepherded America through the Depression } \\
\text { and World War II, then became a symbol of } \\
\text { postwar domestic stability (Gavin, 2018) }\end{array}$ \\
\hline Crooners \\
Crooners & $\begin{array}{l}\text { Radio allowed [the crooner] to create a sense } \\
\text { of intimacy with his listeners by evaporating } \\
\text { the physical boundaries between the performer } \\
\text { and the audience (McCracken, 1999) } \\
\text { Crooners are defined by their intimacy with } \\
\text { as microphone and...crooning is constructed } \\
\text { identification on the part of the individual } \\
\text { listener (McCracken, 1999) }\end{array}$ \\
\hline
\end{tabular}

\section{References}

Andersen, J. (2015). Now you've got the shiveries: Affect, intimacy, and the ASMR whisper

community. Television \& New Media, 16(8), 683-700.

https://doi.org/10.1177/1527476414556184

Barnes, K. (Host). (2001, December 29). Bing Crosby meets [Audio podcast episode]. In Archive on 4. BBC. https://www.bbc.co.uk/sounds/play/b09jvgph

Barratt, E. L., Spence, C., \& Davis, N. J. (2017). Sensory determinants of the autonomous sensory meridian response (ASMR): Understanding the triggers. PeerJ, 5, e3846. https://doi.org/10.7717/peerj.3846

Caballo, V. E., Arias, B., Salazar, I. C., Irurtia, M. J., Hofmann, S. G., \& CISO-A Research Team. (2015). Psychometric properties of an innovative self-report measure: The Social Anxiety Questionnaire for adults. Psychological Assessment, 27(3), 997-1012. https://doi.org/10.1037/a0038828

Crozier, S. (2019, October 24). Billie Eilish and the end of genre. Untitled Magazine. http://untitledmagazine.com/billie-eilish-and-the-end-of-genre

Connick, T. (2019, April 18). How Billie Eilish employed principles of ASMR in her spine-tingling horror-pop. NME. https://www.nme.com/blogs/nme-blogs/billieeilish-asmr-sounds-2478496

Gavin, J. (2019, November 28). How Bing Crosby changed the course of pop music. New York Times.

https://www.nytimes.com/2018/11/28/books/review/gar y-giddins-bing-crosby-biography.html

Geaghan-Breiner, M. \& Gmoser, J. (2019, November 25). How New Artist of the Year, Billie Eilish, harnesses the power of ASMR in her music. Insider.

https://www.insider.com/how-billie-eilish-harnessesthe-power-of-asmr-2019-10

Gosling, S. D., Rentfrow, P. J., \& Swann Jr, W. B. (2003). A very brief measure of the Big-Five personality domains. Journal of Research in Personality, 37(6), 504-528. https://doi.org/10.1016/S0092-6566(03)00046-1

Hammar, P. (1989). Jack Mullin: The man and his machines. Journal of the Audio Engineering Society, 37(6), 490-512.

John, O. P., \& Srivastava, S. (1999). The Big-Five Trait Taxonomy: History, measurement, and theoretical perspectives. In L. A. Pervin \& O. P. John (Eds.), Handbook of personality: Theory and research, Vol.2 (pp. 102-138). New York: Guilford Press.

Kovacevich, A., \& Huron, D. (2019). Two studies of Autonomous Sensory Meridian Response (ASMR): The relationship between ASMR and music-induced frisson. Empirical Musicology Review, 13(1-2), 39-63. https://doi.org/10.18061/emr.v13i1-2.6012

McCracken, A. (1999). "God's gift to us girls": Crooning, gender, and the re-creation of American popular song, 1928-1933. American Music, 17(4), 365-395. https://doi.org/10.2307/3052656

Ollen, J. E. (2006). A criterion-related validity test of selected indicators of musical sophistication using expert ratings (Doctoral dissertation). The Ohio State University, Columbus, $\mathrm{OH}$.

Ordway, S. (2020, May 13). This Friday at 12PM EDT I'm launching a new series of compositions called "Interiors" [Status update]. Facebook. https://www.facebook.com/profile.php?id=17701532

Petrusich, A. (2019, August 23). The Loneliest Generation embraces Billie Eilish. The New Yorker. https://www.newyorker.com/culture/culturalcomment/the-loneliest-generation-embraces-billie-eilish 
Rentfrow, P. J., Goldberg, L. R., \& Levitin, D. J. (2011). The structure of musical preferences: A five-factor model. Journal of Personality and Social Psychology, 100(6), 1139-1157. https://doi.org/10.1037/a0022406 